\title{
Observation and comparative analysis of proton beam extraction or collimation by different planar channels of a bent crystal
}

\author{
A. G. Afonin, V. T. Baranov, M. K. Bulgakov, Yu. A. Chesnokov, P. N. Chirkov, V. N. Gorlov, I. V. Ivanova, \\ D. M. Krylov, A. N. Lun'kov, V. A. Maisheev, S. F. Reshetnikov, D. A. Savin, E. A. Syshchikov, \\ V. I. Terekhov, I. S. Voinov, and I. A. Yazynin \\ Institute for High Energy Physics, 142281, Protvino, Moscow Region, Russia \\ V. B. Ganenko, I. V. Kirillin, N. F. Shul'ga, and V. I. Truten' \\ National Science Center "Kharkov Institute of Physics and Technology", 61108, Kharkov, Ukraine
}

(Received 14 May 2012; published 24 August 2012)

\begin{abstract}
In the experiment the efficiency of the $50 \mathrm{GeV}$ proton beam extraction from accelerator by means of a bent crystal as a function of crystal orientation was measured. This allowed one to make a comparative analysis of efficiencies of high-energy protons deflection by different crystal atomic planes with different values of the electrostatic field. The results of simulation of high-energy protons deflection by means of crystal atomic planes and crystal atomic strings are also presented in the article. In the case of planar channeling the simulation shows a good agreement with experimental data. In the case of proton motion in the regime of stochastic scattering by bent atomic strings the simulation shows that angles of particle deflection are much greater than the critical channeling angle.
\end{abstract}

DOI: 10.1103/PhysRevSTAB.15.081001

PACS numbers: $61.85 .+\mathrm{p}, 29.27 . \mathrm{Eg}$, 07.05.Tp

\section{INTRODUCTION}

Until recently, extraction/collimation of the beam from accelerators was carried out by crystal atomic planes (111) or (110) [1-3]. This mechanism of beam deflection can be realized for positively charged particles for both large and small values of angle $\psi$ between incident particle momentum and the axis of the crystal atomic strings that form the crystal atomic planes. With decreasing angle $\psi$, however, this mechanism of beam deflection collapses. This collapse takes place if the value $\psi$ is a few values of the critical angle of axial channeling $\psi_{c}$. However, such decrease of $\psi$ opens up new possibilities in the particle beams deflection associated with multiple particle scattering on the bent crystal atomic strings and the phenomenon of axial channeling. It is important to note that the axial potentials of the atomic strings are about 5 times higher than the planar potentials. The high axial potential of the crystal field (in $\mathrm{Si}$ potential barrier for proton is higher than $100 \mathrm{eV}$ ) may be used for charged particle beam deflection in the regime of stochastic deflection proposed in [4,5] and experimentally observed in [6]. This mechanism (stochastic mechanism of particle beam deflection by a bent crystal) is due to the multiple particle scattering on the crystal atomic strings in conditions when collisions with different atomic strings may be considered as uncorrelated. In such conditions the phenomenon of dynamical chaos in particle motion in the

Published by the American Physical Society under the terms of the Creative Commons Attribution 3.0 License. Further distribution of this work must maintain attribution to the author(s) and the published article's title, journal citation, and DOI. periodic field of atomic strings occurs [7]. The term "stochastic" here means that the periodicity of crystal atomic strings location is not required for particle deflection. In other words, multiple scattering in the periodic field of bent atomic strings in the considering case practically does not differ from multiple scattering on bent crystal atomic strings which axes in transverse plane are located randomly (random string approximation $[7,8]$ ).

In the spring 2011 run of the accelerator U-70 of the IHEP accelerator complex (Protvino, Moscow Region, Russia) we used several crystals oriented near the crystallographic axis to reflect charged particles by several crystal atomic planes [9]. In the next accelerator run in autumn 2011 we began a systematic study of the efficiencies of the extraction/collimation of the beam from the accelerator by the bent crystal using the planar channeling in a bent crystal and the stochastic deflection mechanism. The first phase of these studies has been associated with carrying out a comparative analysis of the efficiency of beam deflection/collimation by various planar channels of a crystal in conditions of small angles of particle incidence on the crystal with respect to one of the crystallographic axes, when particles can be captured in the planar channeling regime. The results of this phase of the research are described in this paper.

\section{DESCRIPTION OF THE EXPERIMENT}

In the experiment a bent silicon crystal was used for a proton beam extraction from the accelerator. The crystal was placed in the double-axis goniometer and mounted in the ring of the U-70 (Fig. 1). The crystal was oriented near 

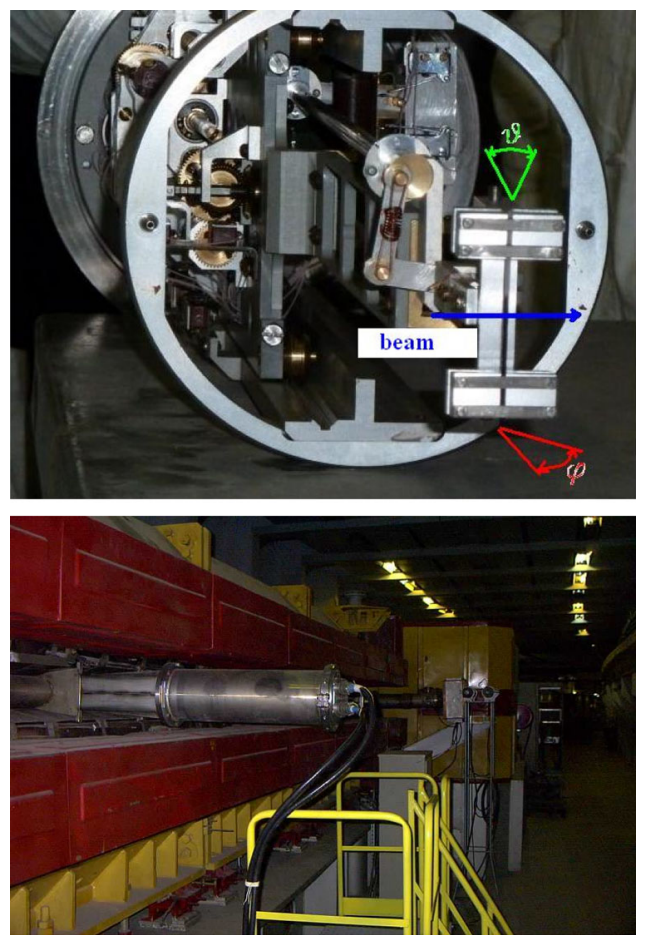

FIG. 1. The crystal in the double-axis goniometer is set in the ring of U-70.

the $\langle 110\rangle$ crystal axis with an accuracy of a few milliradians with the help of a laser device. The sizes of the crystal were $2 \mathrm{~mm}$ along the beam and $0.5 \mathrm{~mm}$ across the beam. The bending angle was $0.7 \mathrm{mrad}$. These crystal parameters were chosen in accordance with the recommendations mentioned in [10] to satisfy the conditions of the dynamical chaos regime in particle motion in the crystal [7,11], otherwise the particles could be captured in planar channels $[8,12]$. In this phase of the experiment, however, the full conditions for the realization of the stochastic mechanism could not be implemented because the angles of orientation of the crystal axis relative to the incident beam were significantly greater than the critical angle of axial channeling. At the same time the possibility of particle capture in various planar channels at the entrance to the crystal was realized in the experiment.

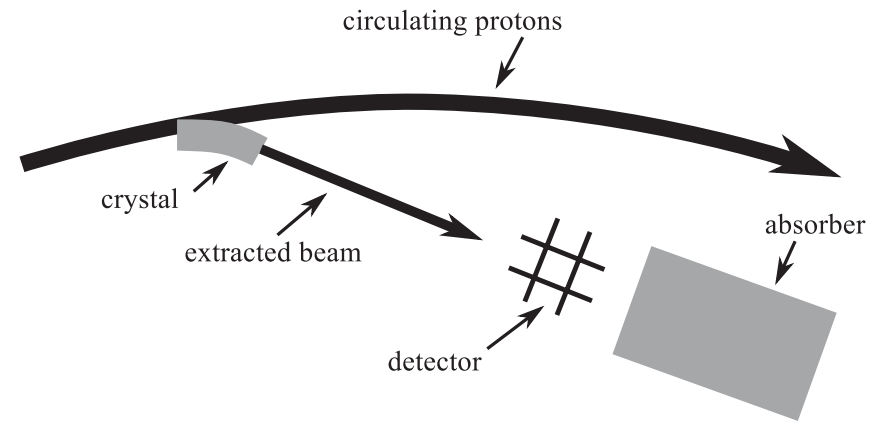

FIG. 2. The experimental setup for the beam extraction and collimation by bent crystals.

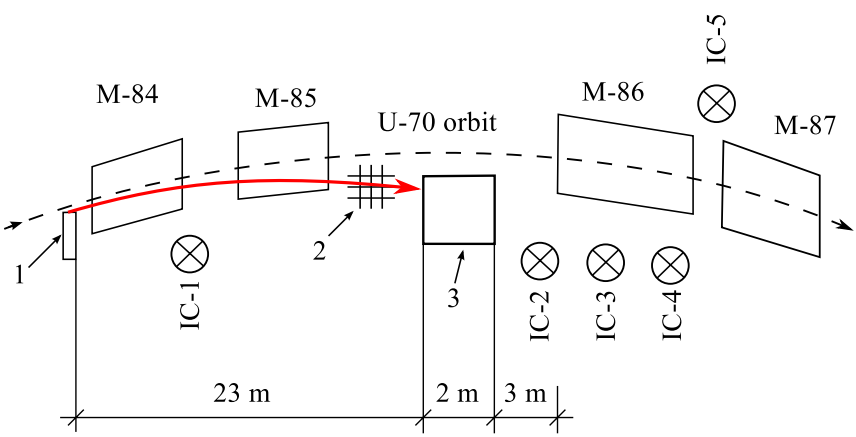

FIG. 3. The main elements of the experimental equipment: 1-station with a crystal, 2-profilometer, 3-absorber, ICionization chambers, $\mathrm{M}-$ magnetic blocks of the accelerator.

In the experiment the crystal was moved in the halo of circulating beam of $50 \mathrm{GeV}$ protons, as shown in Fig. 2, with the help of the remote control system, which allowed one to change the crystal radial position, horizontal and vertical angles of the crystal orientation relative to the circulating beam. Then the beam was gradually directed at the crystal by the slow increasing of the horizontal distortion of protons orbit due to changing magnetic field in the special additional coils of the dipole magnets. The crystal bending angle was large enough to separate circulating and deflected beams. The angle of the beam deflection, the extraction efficiency, the vertical and horizontal distributions of the deflected beam were measured by profilometers (secondary emission detectors) that were located before the absorber in the vacuum chamber of the accelerator near the circulating beam. The parameters of the accelerator, particle detector, the mechanism of the crystal bending, and other experimental details are described in $[13,14]$.

The main elements of the experimental equipment are shown in Fig. 3. The quality of the collimation system was defined by ionization chambers located after the absorber. With the help of the ionization chamber IC-1, which was located near the target, we could estimate the energy loss on the crystal. The minimum of the energy loss determines the optimal angular orientation of the crystal. The rest of the ionization chambers measured the radiation fields, arising in the absorber and in the nearest facilities. The small angle of bending of the crystal $(0.7 \mathrm{mrad})$ did not allow one to output protons from the mode of circulation on single passage through the accelerator ring; therefore, to provide a single passage of the deflected beam through a profilometer the absorber was used.

Orientation dependence of the efficiency of the beam extraction by different crystallographic planes as a function of the horizontal angle of the goniometer rotation is shown in Fig. 4(a). The identification of the planes [Fig. 4(b)] was carried out using the analysis described in the next section of the article.

The efficiency of the beam extraction in the experiment was determined as a ratio of the number of protons 


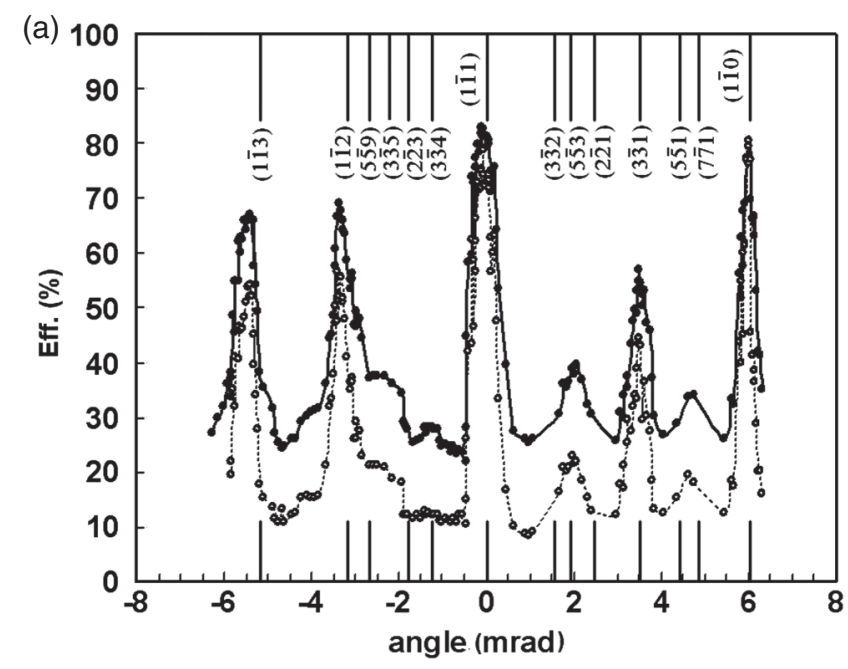

(b)

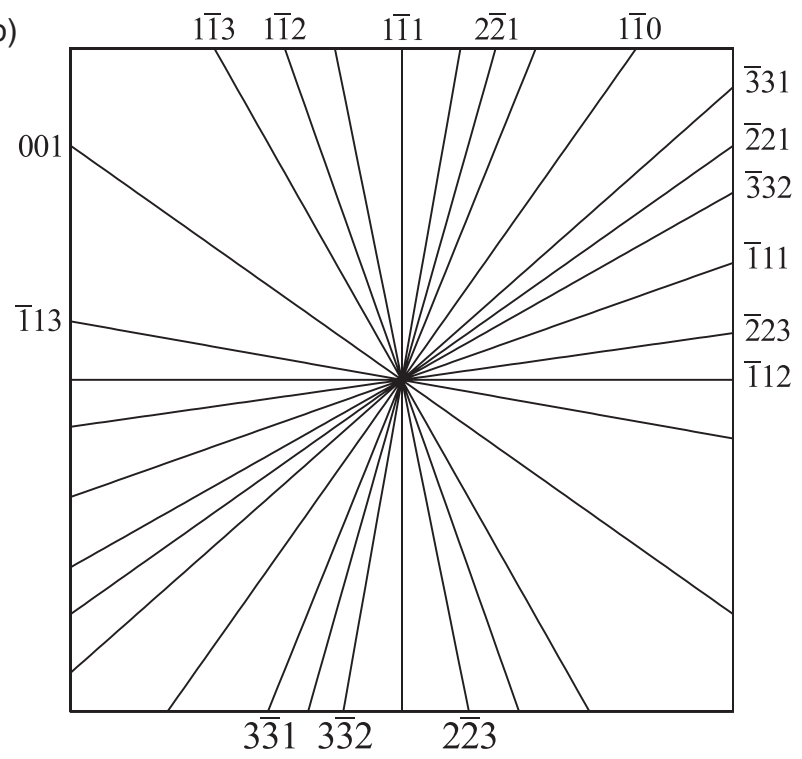

FIG. 4. (a) The efficiency of the accelerated beam extraction as a function of the horizontal angle of the goniometer. The upper curve - the full effect of the deflection due to the planar channeling and incoherent scattering; the lower curve-the contribution of the planar channeling. (b) Stereogram of the most strong atomic planes around the $\langle 110\rangle$ crystal axis of the silicon crystal.

registered by the profilometer to the total number of protons accelerated in the U-70 (measured by means of current transformers). This efficiency is presented by the upper curve in Fig. 4(a). Deflected particles distribution at the particle detector (profilometer) is represented as a superposition of distribution of the amorphous particles scattering (particles detected close to the profilometer edge) and particles deflected by the crystal planes represented by a Gaussian, whose center lies far from the profilometer edge. The lower curve in Fig. 4(a) shows the contribution of the planar channeling in the deflection efficiency (the number of particles in the Gaussian over the total number of protons accelerated in the U-70). To determine the extraction

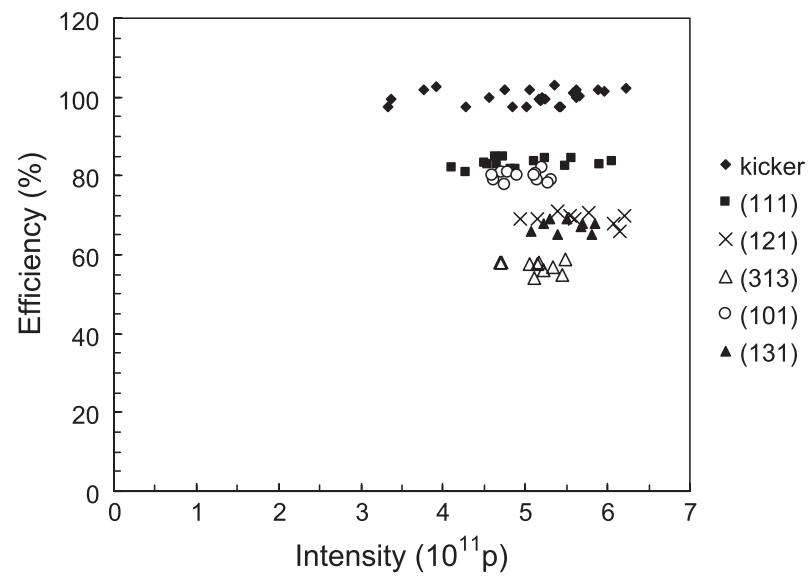

FIG. 5. The comparison of the efficiency of the beam deflection by different crystallographic planes with the extraction by the beam-kicker magnet efficiency.

efficiency the profilometer was calibrated using a fast kicker magnet. Figure 5 shows the sum of the signals from the detector when the beam was deflected by different crystallographic planes in comparison with the effect of the one-turn beam extraction on the detector by the fast kicker
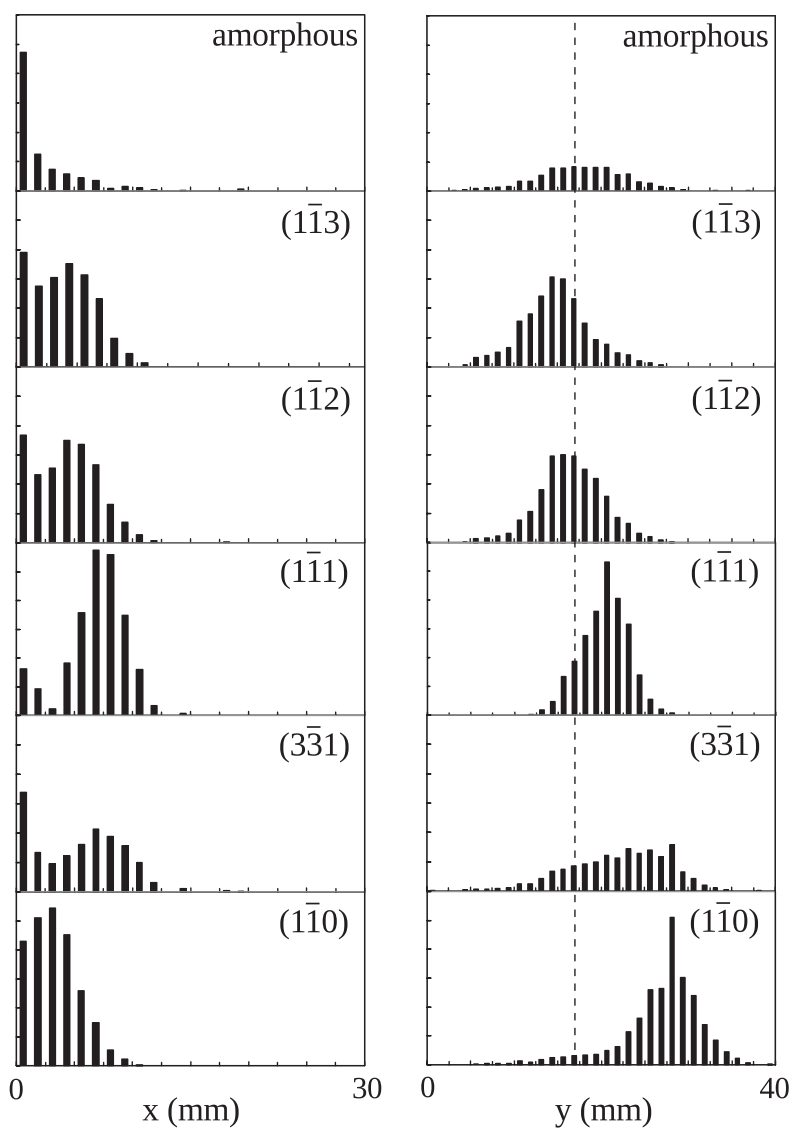

FIG. 6. Beam profiles for different angles of the goniometer [as shown in Fig. 4(a)] corresponding to the deflection by different crystal planes. 
magnet (the whole beam with the size of $6 \mathrm{~mm}$ falls to the aperture of the detector). The measured efficiencies of the extraction for the strong planes (111) and (1110) were $83 \pm$ $3 \%$ and $80 \pm 3 \%$, respectively.

Typical horizontal and vertical profiles of the deflected beam detected by the profilometer are shown in Fig. 6. The left figures show the horizontal beam profiles and the right figures show the vertical profiles. The dashed line in the vertical profiles shows the center of the vertical profile of the beam scattered by the amorphous target.

The data obtained in the first stage of the research allowed us to compare the efficiency of proton beam deflection by crystal planes with different values of the electrostatic field.

\section{COMPARATIVE ANALYSIS OF THE BEAM DEFLECTION BY DIFFERENT CRYSTALLOGRAPHIC PLANES}

In the experiment the efficiencies of the extraction of the proton beam as a function of horizontal angle of the goniometer were measured for the fixed value of the goniometer vertical angle. The results of these measurements are presented in Fig. 4(a) which shows a rather complex behavior of the measured values. Such behavior can be understood if we assume that the observed peaks are due to the protons channeling in the different crystal planes [shown in Fig. 4(b)]. To identify these planes let us consider the motion of a proton in a single crystal (the direction of the particle motion corresponds to the vector $\mathbf{j}$ and is determined by polar angle $\theta$ and azimuthal angle $\alpha$, see Fig. 7).

We chose the reference system in which the $g_{1}$ axis was directed along the $\langle 110\rangle$ crystal axis, $g_{2}$-along the $\langle\overline{1} 12\rangle$ crystal axis and $g_{3}$-along the $\langle 1 \overline{1} 1\rangle$ crystal axis. In the case of ideal crystal fixing relative to the goniometer rotation axes, the horizontal goniometer axis has to be parallel to the $g_{2}$ axis, and the vertical axis has to be parallel to the $g_{3}$ axis. So the relation between the particle

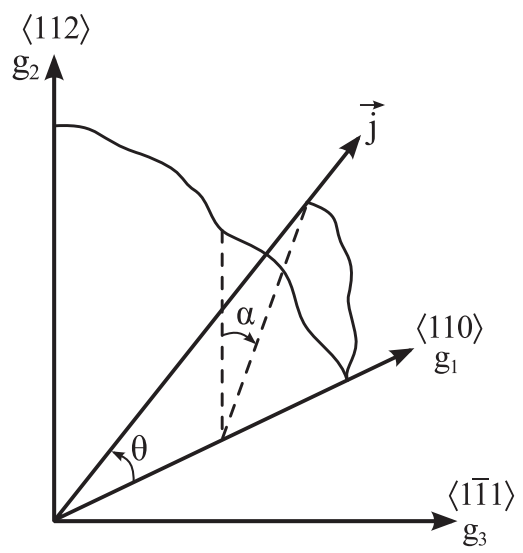

FIG. 7. The particle motion in the system defined by the crystallographic axes of the crystal. angular coordinates and the goniometer horizontal rotation angle should be

$$
\theta_{h}=\theta \sin (\alpha), \quad \theta_{v}=\theta \cos (\alpha) .
$$

Now let us consider the plane that is defined by axes $g_{2}$ and $g_{3}$. There are a lot of crystallographic planes that are orthogonal to the $\left(g_{2}, g_{3}\right)$ plane. All these planes can be used for the particle deflection in the regime of planar channeling. Let us consider one of these planes. Let the angle between this plane and the $g_{2}$ axis be $\beta$. The crystal will be oriented for the channeling in this plane if

$$
\frac{\theta_{h}}{\theta_{v}}=\tan (\beta)
$$

Knowing the $\theta_{h}$ angle from our experiment for one of the crystal planes we can find the $\theta_{v}$ angle.

The experimental data show the presence of two roughly equal efficiency peaks. It can be assumed that the peak at zero angle is due to the channeling in the (11i1) plane, and the peak at an angle of $6 \mathrm{mrad}$ is due to the channeling in the (11̄0) plane. Hence, knowing that $\beta_{1 \overline{1} 0}=0.615479$ radians, we find that

$$
\theta_{v}=\frac{\theta_{h}}{\tan (\beta)}=\frac{0.006}{\tan (0.615479)} \approx 0.0085 \mathrm{rad} \text {. }
$$

Now we can calculate the position of other crystal planes. Comparison of the calculated positions of crystal planes with their experimental positions does not show a very good agreement. For correction of this situation we should taken into account that the goniometric axes can be not absolutely coincident with the crystallographic axes $g_{2}$ and $g_{3}$. As a rule, the results of similar measurements are obtained as a function of horizontal $\left(\theta_{x}\right)$ and vertical $\left(\theta_{y}\right)$ rotation angles around two corresponding goniometric axes. We assume also that both angles are equal to zero when the plane which contains the horizontal and vertical goniometric axes is perpendicular to the $\langle 110\rangle$ crystallographic axis. The connection between $\theta_{h}, \theta_{v}$ and $\theta_{x}, \theta_{y}$ variables is given with the help of the small angle $\gamma$ (see Fig. 8):

$$
\theta_{x}=\frac{\theta_{y} \sin (\beta)}{\cos (\beta-\gamma)} .
$$

There are two unknown parameters $\theta_{y}$ and $\gamma$ in this equation. As a first approximation for $\theta_{y}$ we may take the value of $\theta_{v}$ found above. In this way we obtained a good agreement between the calculated and measured position of the efficiency peaks if $\theta_{v}=0.0088 \mathrm{rad}$ and $\gamma=0.06 \mathrm{rad}$ [see Fig. 4(a)]. A small discrepancy can be explained by errors in the calibration of the goniometer. Note that the planes

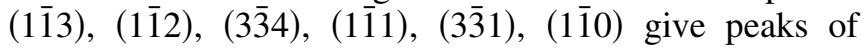
efficiency without the contribution of other planes. The other peaks are the result of the joint influence of two or three neighboring planes that are close enough on particle motion. It is also interesting that the weak planes can 


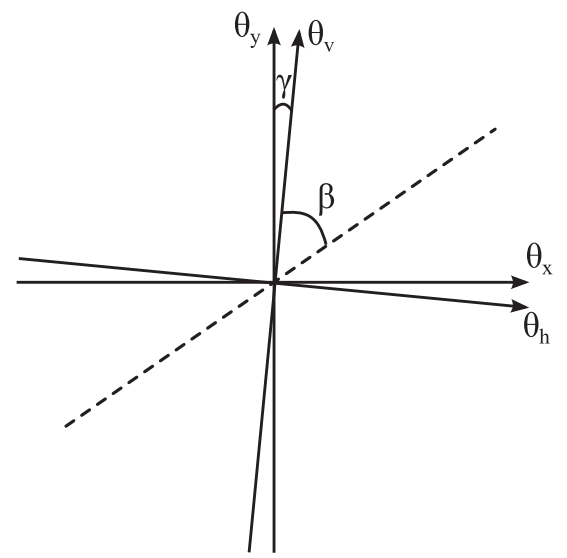

FIG. 8. Azimuthal shift between the coordinate systems of goniometer and crystal. The dashed line shows the plane with an angle $\beta$.

produce quite noticeable efficiency peaks [e.g. the $(3 \overline{3} 4)$ plane, which potential barrier is $1.57 \mathrm{eV}]$.

The potential barriers $U$ of a proton planar channeling in different crystal planes are presented in Table I. The computations were made for potentials calculated according to the work [15] for the atomic form factors obtained from $\mathrm{x}$-ray measurements. Planes with the potential barrier below $0.5 \mathrm{eV}$ have been excluded from the consideration and were not represented in the figure.

Beam deflection by the skew planes (in our crystal orientation it means planes with $\beta \neq 0$ ) is illustrated in Fig. 9. If the crystal size is much smaller than its radius of curvature (this is true for our crystal), one can see that the radius of curvature for the $(\mathrm{klm})$ plane is

$$
R_{(k l m)}=R_{(1 \overline{1} 1)} / \cos (\beta),
$$

where $R_{(1 \overline{1} 1)}$ is the radius of curvature of the (111) crystal plane. The angle of this plane bend is $\phi_{(1 \overline{1} 1)}=d / R_{(1 \overline{1} 1)}$,

TABLE I. Characteristics of crystallographic planes.

\begin{tabular}{lcccccc}
\hline \hline Plane & $\beta$ & $\theta_{x}, \mathrm{mrad}$ & $\theta_{y}, \mathrm{mrad}$ & $U, \mathrm{eV}$ & $\frac{\phi_{x}(\mathrm{klm})}{\phi(111)}$ & $\frac{\phi_{y}(\mathrm{klm})}{\phi(111)}$ \\
\hline$(1 \overline{1} 4)$ & -0.61548 & -6.510 & -6.223 & 3.15 & 0.667 & -0.471 \\
$(1 \overline{1} 3)$ & -0.51480 & -5.162 & -4.978 & 9.16 & 0.758 & -0.429 \\
$(1 \overline{1} 2)$ & -0.33983 & -3.184 & -3.111 & 8.52 & 0.889 & -0.314 \\
$(5 \overline{5} 9)$ & -0.28937 & -2.673 & -2.620 & 1.15 & 0.917 & -0.273 \\
$(3 \overline{3} 5)$ & -0.25167 & -2.232 & -2.263 & 3.16 & 0.938 & -0.241 \\
$(2 \overline{2} 3)$ & -0.19934 & -1.803 & -1.778 & 0.57 & 0.961 & -0.194 \\
$(3 \overline{3} 4)$ & -0.14049 & -1.257 & -1.245 & 1.57 & 0.980 & -0.139 \\
$(1 \overline{1} 1)$ & 0 & 0 & 0 & 22.55 & 1. & 0. \\
$(3 \overline{3} 2)$ & 0.17496 & 1.542 & 1.556 & 2.57 & 0.970 & 0.174 \\
$(5 \overline{5} 3)$ & 0.21423 & 1.893 & 1.915 & 2.49 & 0.955 & 0.208 \\
$(2 \overline{2} 1)$ & 0.27564 & 2.452 & 2.489 & 0.70 & 0.926 & 0.262 \\
$(3 \overline{3} 1)$ & 0.38400 & 3.478 & 3.556 & 5.82 & 0.860 & 0.347 \\
$(5 \overline{5} 1)$ & 0.47499 & 4.398 & 4.525 & 2.79 & 0.791 & 0.407 \\
$(7 \overline{7} 1)$ & 0.51480 & 4.823 & 4.978 & 1.16 & 0.758 & 0.429 \\
$(1 \overline{1} 0)$ & 0.61548 & 5.980 & 6.223 & 21.37 & 0.667 & 0.471 \\
\hline \hline
\end{tabular}

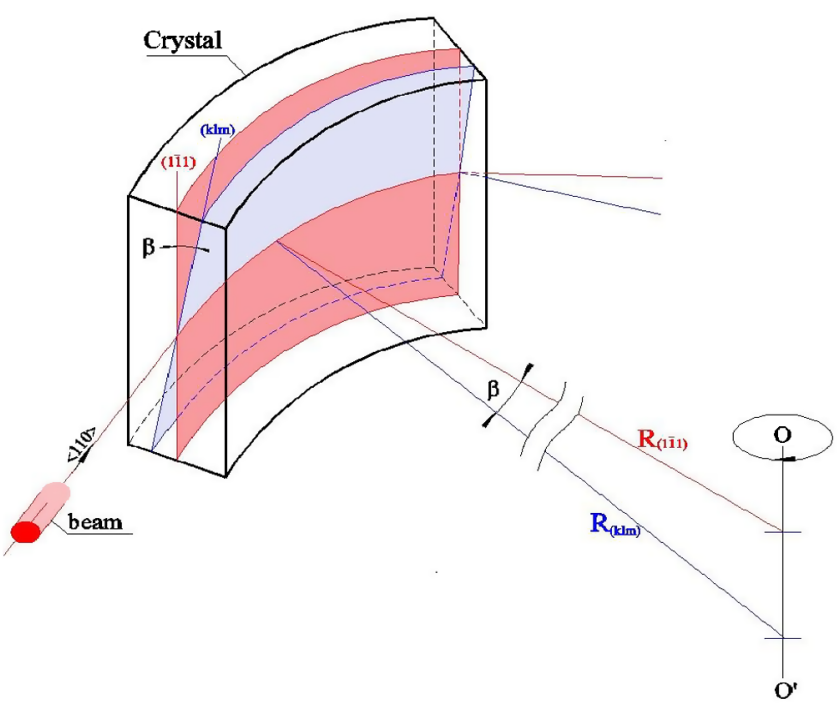

FIG. 9. Beam deflection by the $(\mathrm{klm})$ plane.

where $d$ is the crystal thickness. The bending angle of the $(\mathrm{klm})$ plane for the considered crystal is

$$
\phi_{(k l m)}=\phi_{(1 \overline{1} 1)} \cos (\beta) .
$$

After passing the considered crystal the beam is deflected by the skew plane in the horizontal and vertical directions on angles

$$
\begin{aligned}
& \phi_{x(k l m)}=\phi_{(1 \overline{1} 1)} \cos ^{2}(\beta), \\
& \phi_{y(k l m)}=\phi_{(1 \overline{1} 1)} \cos (\beta) \sin (\beta) .
\end{aligned}
$$

It means that for any orientation the beam is deflected in the same horizontal direction, while the vertical deflection angle can be positive or negative depending on the sign of the angle $\beta$.

In the experiment $\phi_{(1 \overline{1} 1)}$ was approximately $0.7 \mathrm{mrad}$, and $d$ was $2 \mathrm{~mm}$. Consequently, $R_{(1 \overline{1} 1)} \approx 286 \mathrm{~cm}$. The distance between the crystal and the detector was $20 \mathrm{~m}$. However, to calculate the beam deflection angle one should take into account the influence of the gradient of the magnetic field of the accelerator on the particles trajectories. Figure 6 illustrates the deflection of the beam in the horizontal and vertical directions by different crystallographic planes. One can see that the vertical deflection can be in both directions relative to the center of the particle distribution in the case of scattering on an amorphous target.

The extraction efficiency depends on many factors [16], such as parameters of the crystal, characteristics of the proton beam, conditions of the beam operation, and others. In the experiment all these parameters have been fixed and only the horizontal orientation angle of the crystal deflector was changing. So, it was possible to observe the efficiencies of the beam deflection by different crystal planes in approximately the same experimental conditions. In one passage of the proton beam through the crystal deflector, 


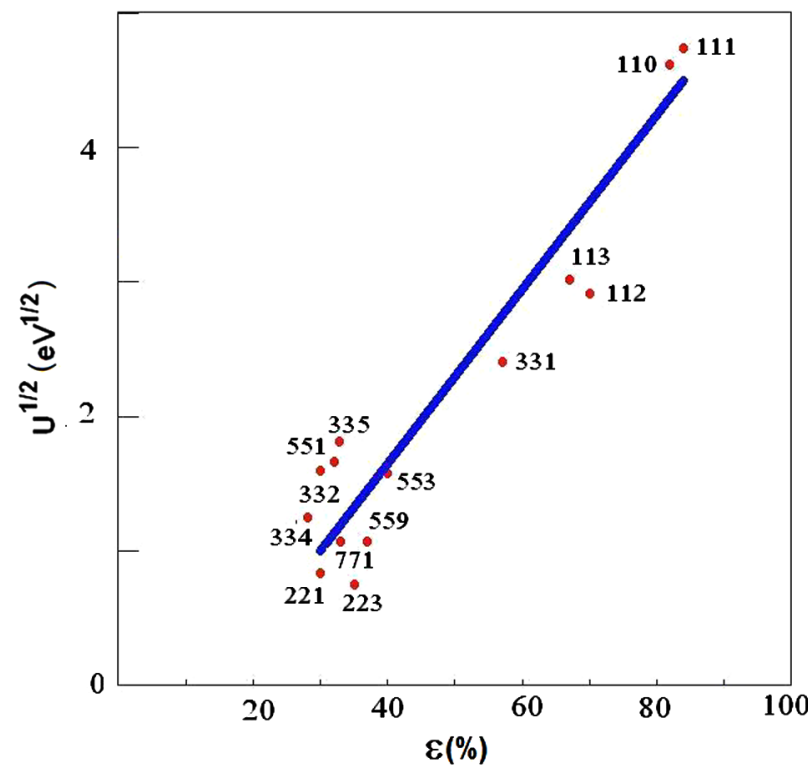

FIG. 10. Linear approximation of the experimental data.

the probability of the particle capture in the channeling mode is approximately proportional to the angular acceptance of the plane (angle of planar channeling, which is proportional to the square root of the potential barrier). However, if the proton beam is circulating in the accelerator, the total number of extracted particles is determined by the number of the proton beam passes through the deflector. Moreover, it should be taken into account that in the result of interaction (mainly due to multiple scattering) in each passage of the beam through the target the geometrical characteristics of the proton beam are changing. Therefore, the linear dependence of the extraction efficiency from the square root of the plane potential barrier is not obvious. Nevertheless, the results of the experiment can be approximately described by the linear dependence (see Fig. 10). When the potential is zero the extraction efficiency is about $15 \%$, which corresponds to the extraction from accelerator by an amorphous target (see Fig. 6).

\section{SIMULATION AND ANALYSIS OF THE EXPERIMENTAL DATA}

\section{A. Planar channeling in a bent crystal}

For a more in-depth analysis of the physical processes taking place in the present experiment the simulation of high-energy protons passage through a bent silicon crystal was carried out. The simulation was performed using the method described in [10]. Using this method the protons angular distribution, horizontal and vertical profiles of the proton beam after passing through the crystal were obtained. The dependence of the efficiency of the proton beam deflection from the bent crystal orientation was also found.

Let us describe some key features of our simulation model. In the simulation $50 \mathrm{GeV} / c$ protons were passing through the bent silicon crystal with thickness of $2 \mathrm{~mm}$ whose bend angle was $700 \mu \mathrm{rad}$. In the experiment the proton beam has an angular divergence of about $0.5 \mathrm{mrad}$, but as the beam was gradually directed at the crystal the angular spread of particles that entered the crystal in each beam cycle was much smaller than the full angular divergence of the beam. As in the simulation we considered single passage of the beam through the crystal, the beam initial angular distribution in the simulation was also much less than $0.5 \mathrm{mrad}$. The initial angular width of the beam in the simulation was chosen from the agreement between the simulation and experimental results for the peak of the efficiency of the beam deflected by the (11i1) plane (the fourth from the top profile in Fig. 6). Such agreement was obtained for the initial angular beam divergence of $20 \mu \mathrm{rad}$. Location of the crystal planes in the simulation was coincident with the location of the crystal planes in the experiment, which is presented in Fig. 4(b). The $\theta_{0 y}$ angle between the horizontal plane (112) and the projection of the initial momentum of a particle on a vertical plane (11 1$)$ in the simulation was $850 \mu \mathrm{rad}$. This angle was 10 times smaller than in the experiment, but since the angle of $850 \mu \mathrm{rad}$ is much greater than the critical angle of axial channeling $\psi_{c}=64.71 \mu \mathrm{rad}$, protons in the simulation, as well as in the experiment, were moving not in the field of separate atomic strings but in the field of crystal atomic planes, so the crystal orientation in the simulation and in the experiment were equivalent. The reduction of the $\theta_{0 y}$ angle allowed us to significantly reduce computing resources required for the simulation.

Figure 11 shows the simulation results for the efficiencies of the proton beam deflection as a function of the initial angle $\theta_{0 x}$ between the vertical plane (11i1) and the projection of the initial momentum of the particle on the horizontal plane (112). In this figure points correspond to the percentage ratio of the number of particles that were scattered in the angular region $\theta_{x}=350-1850 \mu \mathrm{rad}$ to the total number of particles in the beam. Each point corresponds to the simulation results of the beam passage

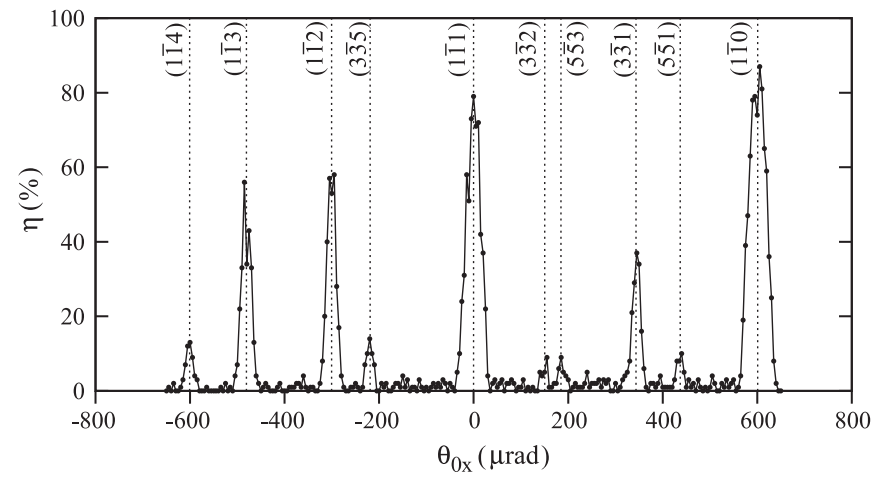

FIG. 11. Simulation results for the efficiency of the proton beam deflection by the bent crystal as a function of the angle $\theta_{0 x} . \theta_{0 y}=850 \mu \mathrm{rad}$. 

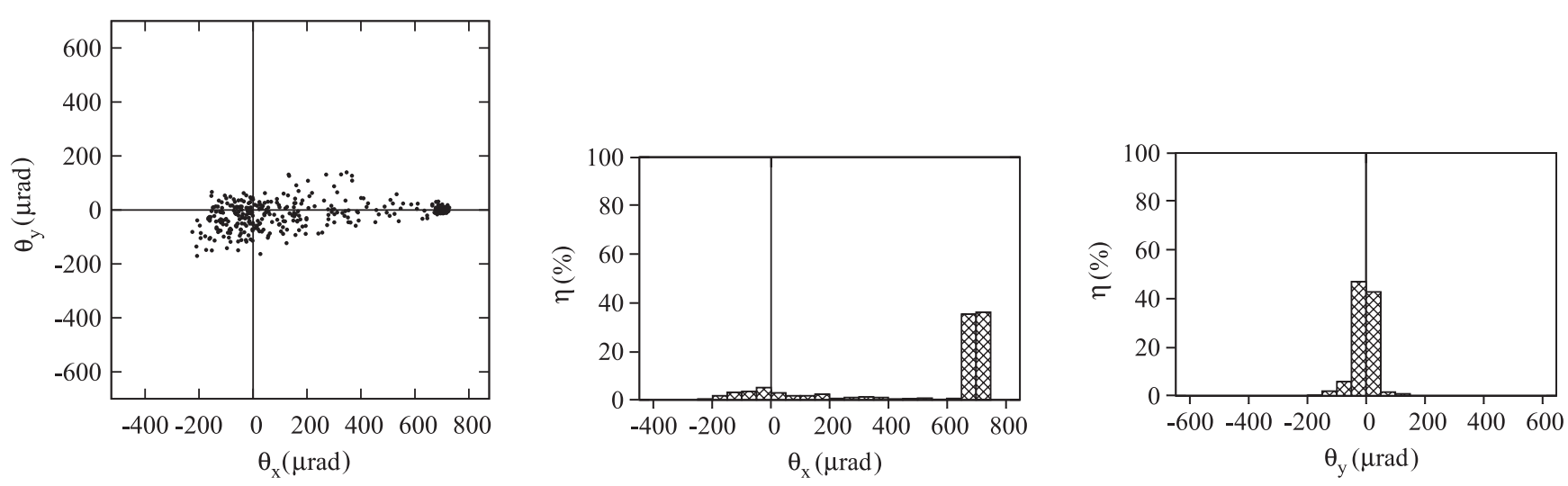

FIG. 12. The angular distribution (left), horizontal (middle), and vertical (right) profiles of the proton beam after passing through the bent crystal. Terms of entrance correspond to the motion of particles in the channeling regime in the vertical plane (111).

through the crystal when the beam incident angle was $\theta_{0 x}$. Scattering in the angular region $\theta_{x}=350-1850 \mu \mathrm{rad}$ indicates particles that entered the detector. Detector width was $30 \mathrm{~mm}$ and it was shifted with respect to the crystal at $7 \mathrm{~mm}$ from the center of the undeflected beam. The angular coordinates of the detector were obtained from the analysis of Fig. 6, where the maximum of the distribution function of particles deflected by the (11) plane was at the point with coordinate $x=7 \mathrm{~mm}$. This maximum corresponds to the deflection of particles by vertical plane (111) at an angle of $700 \mu \mathrm{rad}$. Taking the base as 20 meters, we found that the detector was displaced with respect to the crystal at $7 \mathrm{~mm}$ along the $x$ coordinate. The crystal atomic planes are shown in Fig. 11 by dashed lines.

The results of our simulation show that the peaks of the efficiencies of the protons deflection by means of the bent crystal correspond to the entering of incident particles into vertical or skew planar channels. The efficiency of deflection by low-index planes is much higher than by high-index ones. This is due to the fact that the potential barrier for the channeling of particles in the first case is higher than in the second (see Table I). Figure 11 shows a good agreement between simulation results and experimental results presented in Fig. 4(a). The larger width of the peaks in the experiment is associated with multiturn of the beam, which was not taken into account in the simulation.

Let us consider in more detail the results of simulation of the proton beam deflection by three different crystallographic planes. The case of protons channeling in the vertical plane (111) is shown in Fig. 12. This case corresponds to the initial coordinates of the beam center $\left(\theta_{0 x} ; \theta_{0 y}\right)=(0 ; 850) \mu \mathrm{rad}$. The values $\theta_{x}$ and $\theta_{y}$ in the figure correspond to the angular coordinates of the beam particles, with respect to the initial position of the beam center. The line $\theta_{y}=0$ corresponds to the position of the $(\overline{1} 12)$ plane.

Simulation results show that most of the particles in the beam at the exit from the crystal were moving in the planar channeling regime and were deflected at the angle of crystal bend ( $700 \mu \mathrm{rad})$ in the direction of the crystal bending. The protons that were not captured in the vertical plane channel were either reflected by that plane if their initial angular coordinate $\theta_{x}$ was higher than the critical angle of planar channeling or remained near their initial angular coordinates if the initial angular coordinate $\theta_{x}$ was negative and its absolute value was greater than the critical angle of planar channeling. That is why most of the abovebarrier particles after passing the crystal had a negative angular coordinate $\theta_{x}$.
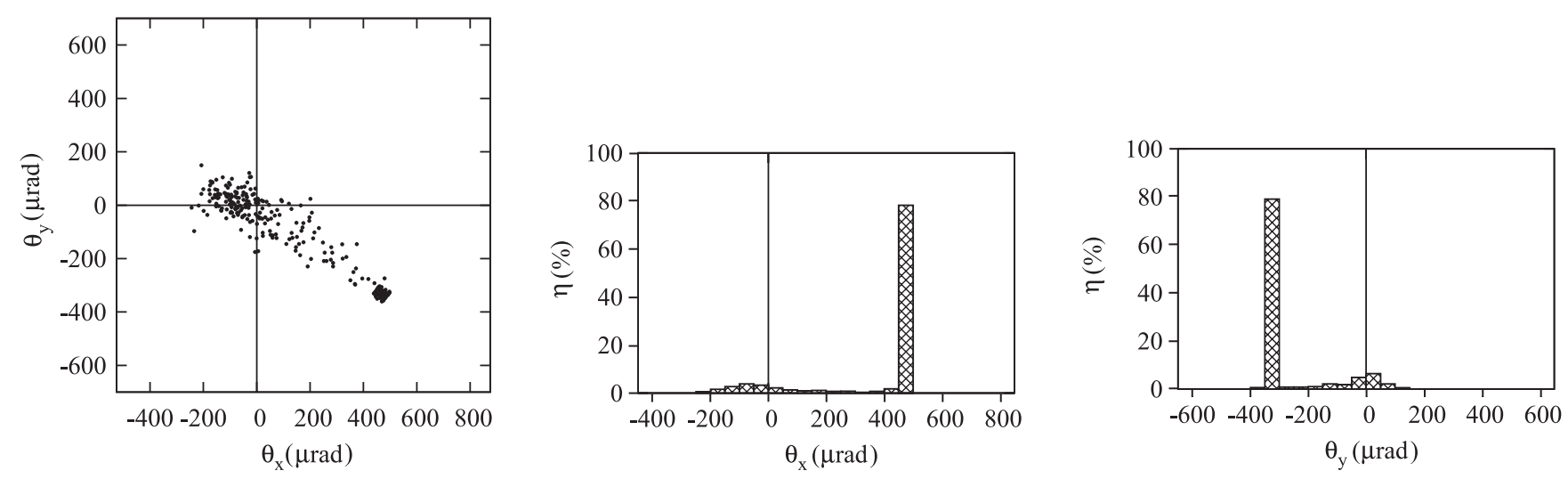

FIG. 13. The same as in Fig. 12 for the conditions of particles motion in the channeling mode in the plane (11̄0). 

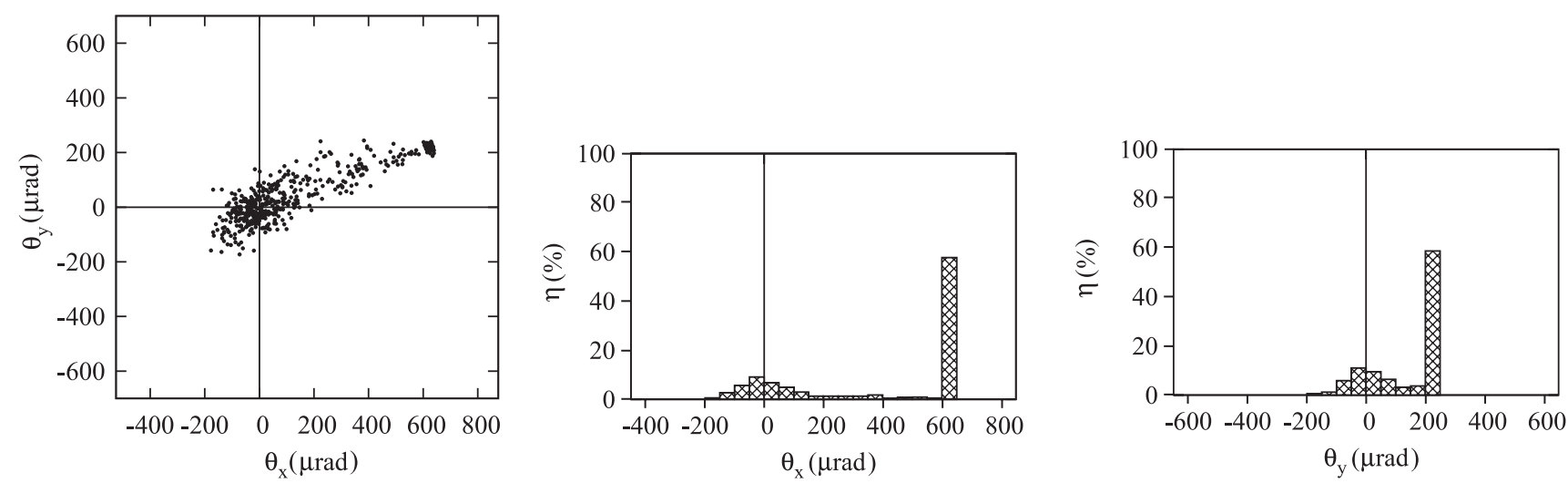

FIG. 14. The same as in Fig. 12 for the conditions of particles motion in the channeling mode in the plane (112).
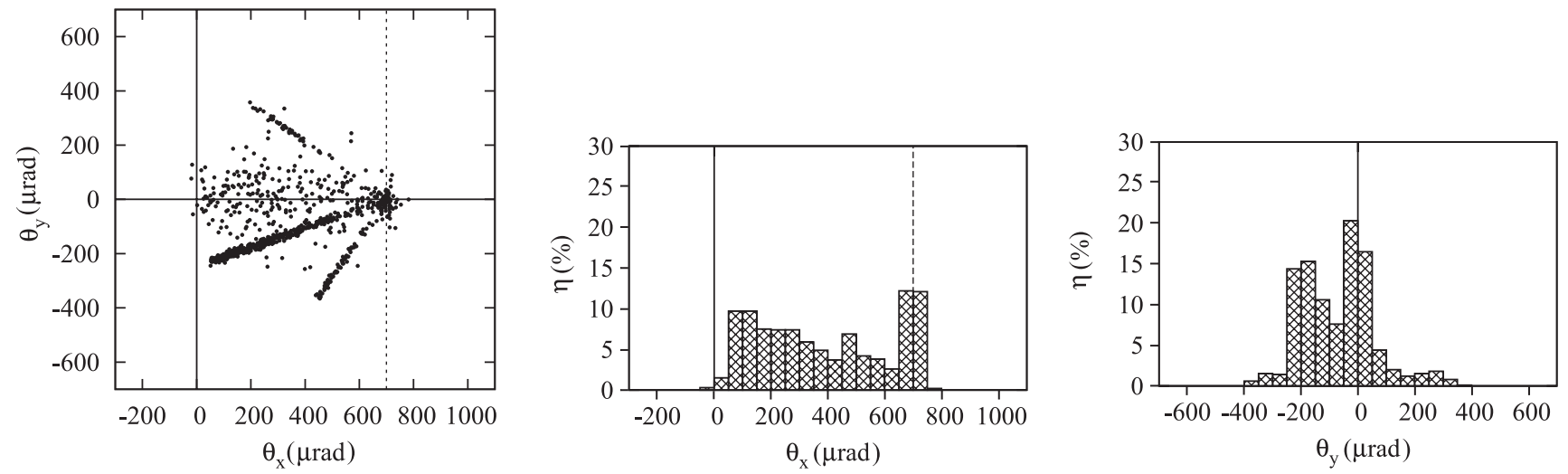

FIG. 15. The same as in Fig. 12 for the conditions of $50 \mathrm{GeV}$ particles motion in the stochastic deflection regime.
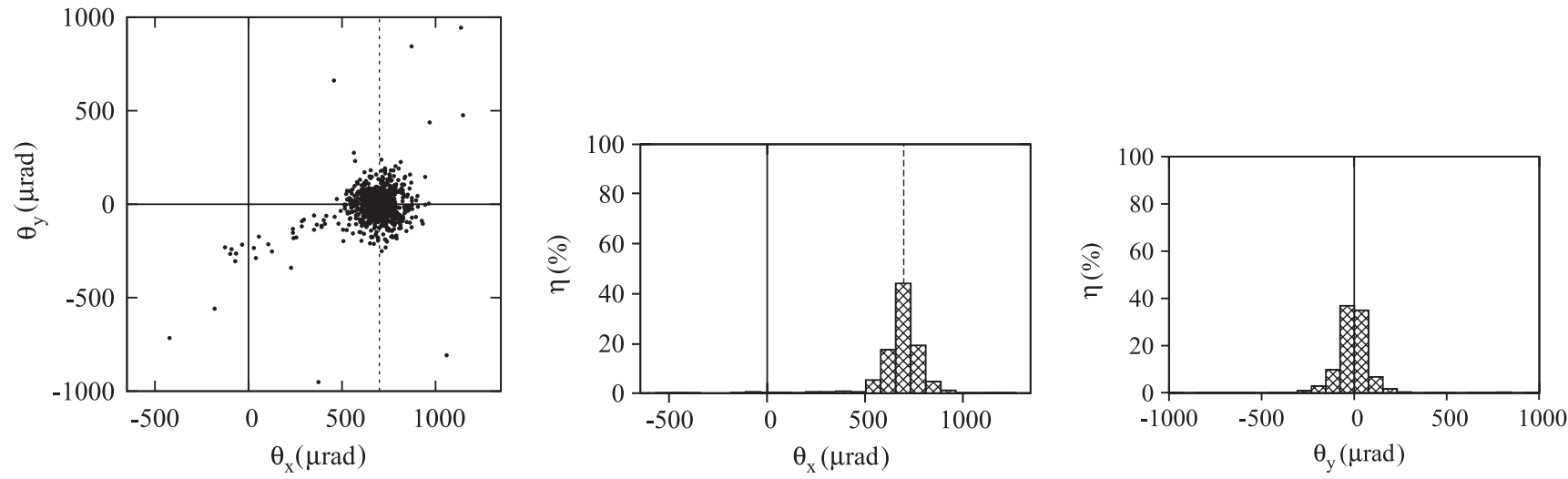

FIG. 16. The same as in Fig. 15 for protons with kinetic energy of $1 \mathrm{GeV}$.

Figure 13 shows similar simulation results for the channeling of the proton beam in the skew plane (1 10$)$. This case corresponds to the initial coordinates of the beam center $\left(\theta_{0 x} ; \theta_{0 y}\right)=(601 ; 850) \mu \mathrm{rad}$. We can see that in addition to the horizontal deflection by the (11) plane protons had the vertical deflection from the initial direction of motion in the direction of the coordinate $\theta_{y}$ decrease. Finally, Fig. 14 shows similar simulation results for the proton beam channeling in the skew plane (11 2$)$. This case corresponds to the initial coordinates of the 
beam center $\left(\theta_{0 x} ; \theta_{0 y}\right)=(-301 ; 850) \mu \mathrm{rad}$. We can see that in addition to the horizontal deflection by the (11) plane protons had the vertical deflection from the initial direction of motion in the direction of the coordinate $\theta_{y}$ increase.

\section{B. Stochastic deflection}

Within the framework of our experiment it is possible to study not only the role of various crystal atomic planes in the deflection of beam particles, but also to study the scattering of particles in a bent crystal in the axial orientation of the crystal relative to the incident beam. Such a study is of considerable interest due to the fact that in this crystal orientation the stochastic deflection by bent crystal atomic strings is possible $[4,5,10]$. The study of this process is planned for the future in the next experimental sessions of U-70. Some of the expected results are illustrated in Fig. 15, which shows the simulation results of $50 \mathrm{GeV}$ protons passage through the bent crystal when particles are incident on the crystal near the $\langle 110\rangle$ crystal axis, i.e., when the initial coordinates of the center of the beam are $\left(\theta_{0 x} ; \theta_{0 x}\right)=(0 ; 0)$. These initial conditions correspond to the motion of particles in the stochastic deflection regime.

The simulation results show that under these initial conditions a significant part of the beam particles follow the bend of the crystal axis, turning to the crystal bending angle $\theta=700 \mu \mathrm{rad}$. These particles are not under barrier. They are moving in the above-barrier regime, multiply scattered by bent crystal atomic strings. Note, however, that the simulation results show that under these conditions along with such particles there are other particles that are trapped in the skew planar channels (111), (110), and (001).

It should be noted that with a decrease of the kinetic energy of the beam particles the part of particles that are trapped in the planar channels under mentioned initial conditions should decrease [5]. In this case the stochastic mechanism of beam deflection by the bent crystal should be performed on the entire thickness of the crystal for almost all of the beam particles. In order to illustrate this, Fig. 16 shows the results of simulation of the protons with kinetic energy of $1 \mathrm{GeV}$ passage through the bent crystal in the same conditions as in Fig. 15. The simulation results show that in this case almost all protons are rotated to the angle of the crystal bend following the bent crystallographic axis. The experimental study of this process is also planned for the next sessions of the experiment.

\section{ACKNOWLEDGMENTS}

This work was supported by the RFBR Grant No. H.4.45.90.11, 1080 (Russia) and by the SFFR Grant No. F40.2/092 (Ukraine).

[1] A. G. Afonin, V.T. Baranov, V.M. Biryukov, M. B. H. Breese, V.N. Chepegin, Yu. A. Chesnokov, V. Guidi, Yu. M. Ivanov, V.I. Kotov, G. Martinelli et al., Phys. Rev. Lett. 87, 094802 (2001).

[2] N. V. Mokhov, G. E. Annala, A. Apyan, R. A. Carrigan, A. I. Drozhdin, T. R. Johnson, A. M. Legan, R. E. Reilly, V. Shiltsev, D. A. Still et al., Int. J. Mod. Phys. A 25, S98 (2010).

[3] W. Scandale, G. Arduini, R. Assmann, C. Bracco, S. Gilardoni, V. Ippolito, E. Laface, R. Losito, A. Masi, E. Metral et al., Phys. Lett. B 692, 78 (2010).

[4] A.A. Grinenko and N.F. Shul'ga, JETP Lett. 54, 524 (1991) [http://www.jetpletters.ac.ru/ps/1265/article_ 19143.shtml].

[5] N. F. Shul'ga and A. A. Greenenko, Phys. Lett. B 353, 373 (1995).

[6] W. Scandale, A. Vomiero, S. Baricordi, P. Dalpiaz, M. Fiorini, V. Guidi, A. Mazzolari, G. Della Mea, R. Milan, G. Ambrosi et al., Phys. Rev. Lett. 101, 164801 (2008).

[7] A. I. Akhiezer, V. I. Truten, and N. F. Shul'ga, Phys. Rep. 203, 289 (1991).

[8] J. F. Bak, P. R. Jensen, H. Madsbøll, S. P. Møller, H. E. Schiøtt, E. Uggerhøj, J. J. Grob, and P. Siffert, Nucl. Phys. B 242, 1 (1984).

[9] A. G. Afonin, V.T. Baranov, M. K. Bulgakov, I. S. Voinov, V. N. Gorlov, I. V. Ivanova, D. M. Krylov, A. N. Lun'kov, V. A. Maisheev, S. F. Reshetnikov et al., JETP Lett. 93, 187 (2011).

[10] N.F. Shul'ga, I. V. Kirillin, and V.I. Truten', Nuovo Cimento C 34, 425 (2011).

[11] A. I. Akhiezer, N. F. Shul'ga, V. I. Truten', A. A. Grinenko, and V. V. Syshchenko, Phys. Usp. 38, 1119 (1995).

[12] A. Baurichter, K. Kirsebom, R. Medenwaldt, S. P. Møller, T. Worm, E. Uggerhøj, C. Biino, M. Clément, N. Doble, K. Elsener et al., Nucl. Instrum. Methods Phys. Res., Sect. B 119, 172 (1996).

[13] A. G. Afonin, V.T. Baranov, V.M. Birjukov, A. A. Kardash, V.I. Kotov, V. A. Maisheev, V.I. Terekhov, E. F. Troyanov, Yu.S. Fedotov, V. N. Chepegin et al., Phys. Part. Nucl. 36, 21 (2005) [http://www1.jinr.ru/ Pepan/2005-v36/v-36-1/3_eng.html].

[14] A. G. Afonin, V. T. Baranov, S. Bellucci, S. A. Belov, S. Bini, V. N. Gorlov, G. Giannini, A. D. Ermolaev, I. V. Ivanova, D. M. Krylov et al., Instrum. Exp. Tech. 54, 1 (2011).

[15] E. Bagli, V. Guidi, and V. A. Maisheev, Phys. Rev. E 81, 026708 (2010).

[16] I. A. Yazynin, V. A. Maisheev, and Yu. A. Chesnokov, Instrum. Exp. Tech. 54, 632 (2011). 